8-1-2020

\title{
Barriers inhibiting detection and management of postpartum hemorrhage by providers in Madagascar
}

Breakthrough RESEARCH

USAID's Health Evaluation and Applied Research Development

Follow this and additional works at: https://knowledgecommons.popcouncil.org/departments_sbsr-rh

Part of the Health Communication Commons, and the Public Health Commons

How does access to this work benefit you? Let us know!

\section{Recommended Citation}

Breakthrough RESEARCH and USAID's Health Evaluation and Applied Research Development. 2020.

"Barriers inhibiting detection and management of postpartum hemorrhage by providers in Madagascar," APPHC Programmatic Research Brief. Washington, DC: Population Council and URC. 


\section{Results}

\section{General insight}

Providers operate in a context of scarcity. While many demonstrate great resourcefulness in addressing structural challenges which impede their work, overcoming these challenges is a concern which is top of mind and likely taxes providers' cognitive bandwidth, therein affecting their ability to respond to and manage complex PPH and other obstetric complications. While providers around the world often struggle with excessive workload, the conditions which these Malagasy providers described suggests that a significant amount of their cognitive bandwidth, ${ }^{4}$ or mental energy, is occupied with seeking ways to overcome basic structural challenges. These challenges range from stock-outs of commodities and medicines and lack of electricity in facilities, to pressure to charge low-income clients for services or convince family members to travel long distances for higher-level care. As one midwife remarked, "There are so many things to do and I don't sleep enough."

\section{Detection and management of PPH}

Insight 1: Due to perceived low PPH prevalence, the risk of other complications are of more concern to providers which leads them to undervalue the importance of strictly complying with preventive measures. Providers frequently mentioned being on the lookout for signs of prolonged labor to refer to a higher level. Given the perceived low probability of PPH compared to other complications, providers may underestimate the importance of strictly complying with $\mathrm{PPH}$ preventive measures, such as oxytocin administration after birth. Many providers cited "GATPA" or Active Management of Third Stage of Labor (AMTSL), particularly oxytocin injection following delivery, as standard procedure; however, our findings suggest inconsistent application of GATPA. When risk of PPH is considered to be low, tradeoffs such as costs to patients may lead providers to selectively follow best practices such as administration of oxytocin, often based on faulty heuristics for risk.

Insight 2: It is difficult to know if oxytocin is given within the one minute window after birth of the baby, and the difference between giving oxytocin within one minute and soon after is not salient, causing providers to apply oxytocin later without knowing that is the case. When describing the delivery process, no provider specifically mentioned the best practice of administering oxytocin within one minute of birth, yet the various tasks related to immediate newborn care prior to GATPA suggests that this window in practice is much longer than one minute. With little or no exposure to $\mathrm{PPH}$ cases, there are no salient consequences for providers to compare what happens when they administer oxytocin within one minute versus later. Without this feedback, providers have no cue to reconsider their behavior.

Insight 3: Women presenting with PPH are not common; therefore, providers' default assumption is that there will be no PPH. Therefore, they do not systematically estimate blood loss until they have visible cues that tell them otherwise, thus delaying detection and management. Most providers have seen few cases of PPH in practice, making them assume there will be no PPH. They also expect that clear and obvious signs would tell them if a woman has PPH. However, providers often cited challenges in measuring blood loss or did not seem to consider measuring blood loss systematically at all: "We just evaluate it from our eyes. There is nothing to use to measure it correctly." Instead of systematically assessing blood loss, providers rely on excessive blood on white fabric as the visual cue that PPH was present, thus potentially delaying detection.

Insight 4: The mental model ${ }^{5}$ that providers have of hemorrhaging is that of rapid, extreme blood loss, therefore providers may discharge patients with slow, continuous blood loss without diagnosing them with PPH. Both the experiences and expectations of bleeding that providers described reflect the mental model that PPH requires rapid, extreme blood loss, and when probed hardly anyone could recall having seen or heard of a case where the bleeding was slow but continuous. In a context where patients and families may be eager to leave the facility and there is pressure on providers to free up limited space and beds, providers may assume patients are fine after a straightforward delivery and discharge early. This tendency to assume that PPH may not be present leads to delays in PPH detection and prompt management and could result in missed cases in the community.

Insight 5: Providers may rely on family members or patients to tell them if there is bleeding rather than monitoring it themselves, especially if they are working alone or the delivery is late at night, which may delay detection. Lack of consistent postpartum monitoring may lead to delays in $\mathrm{PPH}$ detection. A supervisor in Tana remarked of the CSBs, "The patient is there and they [the staff are] going to sleep for some time. They come back, and then they discover [PPH]." When providers are busy and not able to check on clients regularly post-delivery or believe that it isn't necessary, they may rely on patients or their families to tell them if there is bleeding. Despite recognizing the risk of self-report, exhausted midwives may want to convince themselves that the likelihood of complications is low, or if they are operating in a situation of scarcity ${ }^{3}$ they may tunnel on the most salient risks-likely to be other women in labor rather than the woman who has already delivered. 
Insight 6: Even if trained in PPH management, the small number of cases a given provider may attend to makes it easy to forget what one should do, especially since most facilities have no easily accessible, visual reminders of clinical procedures (or they may not be intuitive for providers to understand), or anyone to ask in the moment of action. Most providers interviewed had seen very few cases of PPH outside of their training, and had difficulty describing the steps they would take to manage a case. Given this low number of cases they have managed and the variation in treatment protocols depending on the source of $\mathrm{PPH}$, it is unlikely that providers will recall what to do in the moment without assistance of some kind. Although several doctors reported referring to books, in a situation where bleeding is heavy and the provider is alone, it is difficult to envision how a provider would easily refer to a book to know how to proceed. As a result, it is likely that providers simply follow their intuition as to what to do. Also, rural providers are likely to be working alone without direct supervision, requiring them to make decisions on their own.

Insight 7: There is a mismatch between practices providers are trained on and what is feasible in the facilities, leaving providers to seek ways to adapt best practices according to their own experience or on the basis of what others in the facility tell them should be done. Providers receive training on best practices for managing PPH but get little experience applying them in practice. Providers described needing to adapt their practice to the equipment constraints of rural facilities, or to the habits of others at the facility who may be more senior, even if they were less recently trained. One trainee midwife described, "There are times when you feel like you master things, but when you think of the Major or the Chief, they tell you they are elders and experienced, that you have to follow them, [even] though there might be something wrong."

Insight 8: Essential commodities and medicines required to manage PPH are not available in facilities, therefore providers read the leaflets of medicines they have on hand to determine what they could potentially use. For example, some providers use Vitamin K1 for PPH management since one of the medical indications mentions blood clotting (albeit for newborns). In the face of uncertainty in difficult situations, providers may rely on materials at hand for determining how to treat certain conditions, which may be misaligned with best practices. The frequency with which K1 was mentioned as a method to manage PPH suggests trainees and potentially other providers rely on medical leaflets as a means to see which medicines they have on hand could be used for different cases they face. Tranexamic acid was not a treatment that providers had heard of.

\section{Other insights}

In a country where most births do not take place in a facility, women's decision to deliver at home and the detection and referral of PPH during home births both affect the extent to which facility-based provider behavior can impact mortality outcomes. Although these other behaviors were not fully explored in this study, we note below a few emerging insights related to the experiences of postpartum women and TBAs. Our recommendation is that additional research is needed to build on these initial findings more conclusively.

Insight 1: There are many tradeoffs implied in delivering at a facility and in the context of scarce resources, delivering at home may be the most rational choice for many women.

Insight 2: Delivering in a facility requires prior planning and women do not plan in this way, which may be compounded by fear of what may happen if others know the delivery date.

Insight 3: Facilities and medical equipment may feel "foreign" and intimidating to women, which may be further compounded by feelings of shame around their socioeconomic status or fear of mistreatment.

Insight 4: In a context where institutional healthcare access has been limited, cultural narratives have been constructed which explain complications in a way that puts in question the usefulness of delivering in a facility since these challenges would not be effectively addressed there.

Insight 5: TBAs focus primarily on delivering a healthy baby and may leave the woman's home soon after delivery or not note bleeding immediately while they care for the child, which may lead to delayed detection.

Insight 6: TBAs do not always ensure placenta completeness and there is often some bleeding as a result. TBAs are not concerned with bleeding that is not extreme and may attribute it to a normal process of "cleansing."

Insight 7: Resistance from the woman or her family to be referred, overconfidence in her ability to manage complications, concern about the repercussions, or inability to locate timely transportation may lead TBAs to try to manage PPH cases themselves even if they know they should refer the woman to a hospital.

Insight 8: PPH risk is not top of mind for TBAs and PPH often does not have warning signs, therefore TBAs wait for a complication that they cannot manage before deciding to refer, causing delays and challenges in referring the woman to a hospital. 


\section{Conclusion}

Together, these insights highlight the need for innovative solutions to address the behavioral barriers providers face in complying with best practices to detect and manage $\mathrm{PPH}$, particularly in the light of the challenging circumstances under which they work. Designing to change or adjust for specific elements in the context of providers can help to eliminate or decrease the effect of these behavioral barriers. For instance, providing real-time support using enhanced visual aids for how to manage infrequently seen complications or by providing remote support as an extension of mentorship could be more effective than training efforts which are distant from the moment of emergency and not practically applicable to their working context.

Collaborative co-creation processes and iterative testing will ensure that new interventions are best positioned for impact and are specific to and responsive to the needs of providers and women in labor. This approach to intervention design has already been successfully used to develop solutions related to provider behavior, such as to enhance respectful maternal care in Zambia and improve malaria screening and treatment in Nigeria, and could hold great promise for enhancing the quality of PPH care in Madagascar.

\section{References}

1. Say, L. et al. 2014. "Global causes of maternal death: a WHO systematic analysis," Lancet Global Health 2(6): e323-e333. doi:10.1016/ S2214-109X(14)70227-X

2. Rakotozanany, B. et al. 2019. "Maternal mortality related to postpartum hemorrhage: a case-control study at the Befelatanana maternity of Madagascar," International Journal of Reproduction, Contraception, Obstetrics and Gynecology 8(1): 121-126. doi: 10.18203/2320-1770.ijrcog20185406

3. UNICEF. 2014. State of the World's Children 2015. Geneva: UNICEF.

4. Mullainathan, S., E. Shafir. 2013. Scarcity: Why Having Too Little Means So Much. New York: Macmillan.

5. Norman, D.A. 1983. "Some observations on mental models." In Dedre Gentner, Albert L. Stevens (eds) Mental Models. New York: Psychology Press, 15-22.

This work is part of the Advancing Postpartum Hemorrhage Care (APPHC) partnership supported by USAID and led by the Breakthrough RESEARCH Project and the Health Evaluation and Applied Research Development (HEARD) Project. The APPHC partnership generates and tests solutions to address key implementation barriers for PPH prevention and treatment and contributes to the effective implementation of interventions, strategies, and innovations for PPH in Madagascar and Malawi.

https://www.respectfulcareresources.com/apphc

\section{Acknowledgments}

This research brief describes work led by ideas 42 under Breakthrough RESEARCH in collaboration with ACCESS and the District Health Offices of Manakaran and Vohipeno. We are also grateful for the support of all midwives, women, and other interview participants who agreed to speak with us during this work.

\section{Suggested citation:}

Breakthrough RESEARCH and USAID's Health Evaluation and Applied Research Development. 2020. "Barriers inhibiting effective detection and management of PPH by providers in Madagascar," APPHC Programmatic Research Brief. Washington DC: Population Council and URC.

Front cover photo credit: CMaddie Kau, ideas42

C 2020 The Population Council. All rights reserved.

\section{Breakthrough RESEARCH}

Population Council

4301 Connecticut Ave., NW

Suite 280

Washington, DC 20008

+1 2022379400

breakthroughactionandresearch.org

BreakthroughResearch@popcouncil.org
USAID's Health Evaluation and Applied Research Development

\section{URC}

5404 Wisconsin Ave

Suite 800

Chevy Chase, MD 20815

www.heardproject.org

heard@urc-chs.com

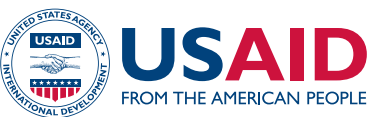

This document is made possible by the generous support of the American people through the United States Agency for International Development (USAID) under cooperative agreements Breakthrough RESEARCH (AID-OAA-A-17-00018) and USAID's Health Evaluation and Applied Research Development Project (HEARD) (AID-OAA-A-17-00002). The contents of this document are the sole responsibility of Breakthrough RESEARCH, Population Council, and University Research Co., LLC and do not necessarily reflect the views of USAID or the United States Government.
USAID'S HEALTH EVALUATION AND APPLIED RESEARCH DEVELOPMENT (HEARD) PROJECT

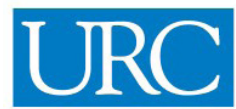

USAID's Health Evaluation and Applied Research Development (HEARD) project leverages a global partnership of more than 30 institutions to generate, synthesize, and use evidence to improve the implementation of policies and programs related to USAID priority areas, and crucial for improving health and development in low and middle-income countries.

\section{(2. POpUlation COUNCIL \\ Ideas. Evidence. Impact.}

Breakthrough RESEARCH catalyzes SBC by conducting state-of-the-art research and evaluation and promoting evidence-based solutions to improve health and development programs around the world. Breakthrough RESEARCH is a consortium led by the Population Council in partnership with Avenir Health, ideas42, Institute for Reproductive Health at Georgetown University, Population Reference Bureau, and Tulane University. 